\title{
HIV induces the expression of activation-induced cytidine deaminase (AICDA) in B cells through a direct interaction between virion-associated CD40L and CD40
}

\author{
M Epeldegui*, S Kitchen, J De La Cruz, J Zack and O Martinez-Maza
}

Address: UCLA AIDS Institute, David Geffen School of Medicine, Los Angeles, California, USA

* Corresponding author

\author{
from I I th International Conference on Malignancies in AIDS and Other Acquired Immunodeficiencies (ICMAOI): Basic, Epidemiologic, and Clinical \\ Research \\ Bethesda, MD, USA. 6-7 October 2008 \\ Published: 17 June 2009 \\ Infectious Agents and Cancer 2009, 4(Suppl 2):OII doi:10.1 186/I750-9378-4-S2-OI I
}

This abstract is available from: http://www.infectagentscancer.com/content/4/S2/O I I

(c) 2009 Epeldegui et al; licensee BioMed Central Ltd.

NHL is a common AIDS-associated cancer. NHL is thought to occur due to errors in Class Switch Recombination (CSR) and Somatic Hypermutation (SHM); both of these events occur in Germinal Center (GC) B cells. Activation-Induced Cytidine deaminase (AICDA) is an enzyme required for both CSR and SHM. Since errors in both CSR and SHM lead to the seminal molecular lesions in NHL development, it is thought that AICDA plays a central role in the genesis of these cancers. It has been shown by us and others that several oncogenic viruses (EBV, HCV, HPV) can induce AICDA expression. We have also shown that AICDA expression in PBMC is elevated prior to AIDS-NHL diagnosis. Additionally, several studies have shown that exposure of B cells to HIV can result in their activation.

Based on this, we assessed the ability of HIV to induce AICDA expression in normal human B cells. We hypothesized that the HIV virion itself might be inducing AICDA expression, through a direct interaction between the virus and $\mathrm{B}$ cells involving the ligation of CD40 on B cells by CD40L. CD40L classically induces AICDA expression in B cells. It is known that CD40L is incorporated into the HIV envelope membrane. We observed that HIV grown in PBMC induces AICDA mRNA expression in B cells and that these virions express CD40L. Additionally, we created HIV that do not express CD40L, as well as HIV that does contain CD40L. Only viruses expressing CD40L induced AICDA expression in B cells. AICDA expression in B cells exposed to viruses expressing CD40L was abrogated by anti-CD40L blocking antibody, but not by exposure to AZT.

In conclusion, HIV viruses that express CD40L on their surfaces induce AICDA expression in B cells, and this induction is due to a direct interaction between the CD40L on these virions and CD40 on B cells. Also, HIV infection of the $\mathrm{B}$ cells does not play a role in this induction. CD40L-expressing viruses induced AICDA expression at both the mRNA and protein levels. These findings are of great interest as they confirm a direct role for HIV in $B$ cell activation, and in the induction of a gene (AICDA) that plays a central role in NHL development. Therefore, HIV has the potential to contribute to NHL development through a direct interaction with $\mathrm{B}$ cells. 